Теорія Ймовір. та Матем. Статист. Вип. 81, 2010
Theor. Probability and Math. Statist. No. 81, 2010, Pages 51-58 S 0094-9000(2010)00809-X

Article electronically published on January 18, 2011

\title{
SOME LIMIT THEOREMS FOR CONTROLLED BRANCHING PROCESSES
}

UDC 519.21

\author{
YA. M. KHUSANBAEV
}

\begin{abstract}
Limit theorems are obtained for branching processes depending on the size of the population. These results contain the case of convergence of such processes to the deterministic limit. Functional central limit theorems for fluctuations are proved.
\end{abstract}

Let $\left\{\xi_{k, j}(l), k, j, l \in \mathbb{N}\right\}$ be a family of nonnegative integer-valued independent random variables. Assume that the random variables $\xi_{k, j}(l), k, j \in \mathbb{N}$, are identically distributed. We define the stochastic process $X_{k}, k \geq 0$, by the following recurrence relation:

$$
X_{0}=1, \quad X_{k}=\sum_{j=1}^{X_{k-1}} \xi_{k, j}\left(X_{k-1}\right), \quad k \geq 1 .
$$

This process is called a branching process dependent on the size of the population or a controlled branching process.

The branching processes dependent on the population size are studied, for example, in the papers [1, 2], 5]-10, [14-[16], 18]-21. Conditions for the extinction of processes (1) as well as those for the convergence of normalized random variables $X_{n}$ to a finite random variable are studied in [2]-[5] and [6, 7, 18]. The central limit theorem for controlled branching processes (11) is proved in [8. Sufficient conditions for the weak convergence as $n \rightarrow \infty$ of the distribution of $X_{n} / n$ to the gamma distribution are found in [16, 21].

In 19 the authors consider a model for a population of cells in which every cell lives for a unit of time and then divides into two or dies, with probability of division depending on the size of the population. Also the asymptotic distribution function for the time when the size of the population exceeds given limits is found there. The results of [19] are extended in 20] to a more general class of stochastic processes. Some statistical problems for the processes of type (1) are studied in 9, 10.

The so-called $\varphi$-controlled branching processes are studied in [14]-15]. In the current paper, we obtain some sufficient conditions for the convergence as $n \rightarrow \infty$ of the processes $\left\{X_{[n t]}, t \geq 0\right\}, n \geq 1$, to a deterministic process (the symbol $[a]$ stands for the integer part of a number $a$ ), as well as functional central limit theorems for the deviation of $X_{[n t]}, t \geq 0$, from the limit process.

In what follows, we always assume that $\mathrm{E} \xi_{1,1}^{2}(x)<\infty$ for all $x \in \mathbb{N}$. We introduce the notation

$$
m(x)=\mathrm{E} \xi_{1,1}(x), \quad \sigma^{2}(x)=\operatorname{Var} \xi_{1,1}(x) .
$$

2000 Mathematics Subject Classification. Primary 60J80; Secondary 60F17, 60J65.

Key words and phrases. Population-size-dependent branching process, weak convergence, Wiener process, limit theorem. 
Let $\mathcal{F}_{k}=\sigma\left\{X_{0}, X_{1}, \ldots, X_{k-1}\right\}$ be the $\sigma$-algebra generated by random variables

$$
\left\{X_{0}, X_{1}, \ldots, X_{k-1}\right\} \text {. }
$$

A process of the form (1) can be represented as

$$
X_{k}=X_{k-1}+\left(m\left(X_{k-1}\right)-1\right) X_{k-1}+M_{k},
$$

where

$$
M_{k}=\sum_{k=1}^{X_{k-1}}\left(\xi_{k, j}\left(X_{k-1}\right)-m\left(X_{k-1}\right)\right) .
$$

It is clear that $M_{k}, k \geq 1$, is a martingale difference with respect to the filtration $\mathcal{F}_{k}$, $k \geq 1$. Consider the random event $\varepsilon_{\infty}=\left\{X_{n} \rightarrow \infty\right\}$ and put $q=1-\mathrm{P}\left(\varepsilon_{\infty}\right)$. In what follows $T>0$ denotes an arbitrary fixed number and $I(A)$ stands for the indicator of a random event $A$.

Theorem 1. Let $m(x)=1+\alpha / x$ for $x>0$ and some $\alpha>0$. Suppose $x \sigma^{2}(x) \leq C x^{\beta}$ for some $0 \leq \beta<1$. Then

$$
\sup _{0 \leq t \leq T}\left|\frac{X_{[n t]}}{n}-\alpha t\right| \stackrel{\mathrm{P}}{\rightarrow} 0 \quad \text { as } n \rightarrow \infty
$$

on the event $\varepsilon_{\infty}$.

Theorem 1 implies, in particular, that $\mathrm{P}\left(\varepsilon_{\infty}\right)>0$. This can be shown by using some results of the paper [4.

Proof of Theorem 1, We have

$$
X_{k+1}=X_{k}+\left(m\left(X_{k}\right)-1\right) X_{k} I\left(X_{k}>0\right)+M_{k} .
$$

Passing to the expectation and then summing in $k$ we obtain

$$
\mathrm{E} X_{n+1}=1+\alpha \sum_{k=0}^{n} \mathrm{P}\left(X_{k}>0\right)
$$

Since $\lim _{n \rightarrow \infty} \mathrm{P}\left(X_{n}>0\right)=1-q$, the latter relation together with the Toeplitz lemma implies that

$$
\mathrm{E} \frac{X_{n}}{n} \rightarrow \alpha(1-q) \quad \text { as } n \rightarrow \infty
$$

Further, relation (2) yields

$$
X_{k+1}^{2}=X_{k}^{2}+2\left(m\left(X_{k}\right)-1\right) X_{k}^{2}+\left(m\left(X_{k}\right)-1\right)^{2} X_{k}^{2}+M_{k}^{2}+2 m\left(X_{k}\right) X_{k} M_{k},
$$

whence

$$
\mathrm{E} X_{k+1}^{2}=\mathrm{E} X_{k}^{2}+2 \alpha \mathrm{E} X_{k}+\alpha^{2} \mathrm{P}\left(X_{k}>0\right)+\mathrm{E} X_{k} \sigma^{2}\left(X_{k}\right) .
$$

Summing these equalities we get

$$
\mathrm{E} X_{n+1}^{2}=1+2 \alpha \sum_{k=0}^{n} \mathrm{E} X_{k}+\alpha^{2} \sum_{k=0}^{n} \mathrm{P}\left(X_{k}>0\right)+\sum_{k=0}^{n} \mathrm{E} X_{k} \sigma^{2}\left(X_{k}\right) .
$$

It is obvious that

$$
\frac{1}{(n+1)^{2}} \sum_{k=0}^{n} \mathrm{P}\left(X_{k}>0\right) \leq \frac{n}{(n+1)^{2}} \rightarrow 0 \quad \text { as } n \rightarrow \infty .
$$


We obtain from (3) that

$$
\frac{2 \alpha}{(n+1)^{2}} \sum_{k=0}^{n} \mathrm{E} X_{k} \sim \frac{2 \alpha^{2}(1-q)}{(n+1)^{2}} \sum_{k=1}^{n} k \rightarrow \alpha^{2}(1-q) \quad \text { as } n \rightarrow \infty
$$

by the Toeplitz lemma.

The assumptions of the theorem and the well-known inequality

$$
\left(\mathrm{E}|\xi|^{r}\right)^{1 / r} \leq\left(\mathrm{E}|\xi|^{l}\right)^{1 / l}, \quad r \leq l,
$$

imply that

$$
\mathrm{E} X_{k} \sigma^{2}\left(X_{k}\right) \leq C \mathrm{E} X_{k}^{\beta} \leq C\left(\mathrm{E} X_{k}\right)^{\beta} .
$$

Taking into account asymptotic relation (3) and applying the Toeplitz lemma again, we prove that

$$
\begin{aligned}
\sum_{k=0}^{n} \mathrm{E} X_{k} \sigma^{2}\left(X_{k}\right) & \leq C \sum_{k=1}^{n}\left(\mathrm{E} X_{k}\right)^{\beta} \sim C(\alpha(1-q))^{\beta} \sum_{k=1}^{n} k^{\beta} \\
& \sim C(\alpha(1-q))^{\beta} \int_{0}^{1} x^{\beta} d x \cdot n^{1+\beta}=C \frac{(\alpha(1-q))^{\beta}}{1+\beta} n^{1+\beta}
\end{aligned}
$$

as $n \rightarrow \infty$. Therefore

$$
\frac{1}{(n+1)^{2}} \sum_{k=1}^{n} \mathrm{E} X_{k} \sigma^{2}\left(X_{k}\right) \leq C \frac{(\alpha(1-q))^{\beta}}{1+\beta} \cdot \frac{n^{1+\beta}}{(n+1)^{2}} \rightarrow 0 \quad \text { as } n \rightarrow \infty,
$$

since $\beta<1$. Thus relations (44)-(6) imply that

$$
\mathrm{E}\left(\frac{X_{n}}{n}\right)^{2} \rightarrow \alpha^{2}(1-q) \text { as } n \rightarrow \infty
$$

We derive from the latter result and (3) that

$$
\frac{X_{[n t]}}{n} \stackrel{\mathrm{P}}{\rightarrow} \alpha t \quad \text { as } n \rightarrow \infty
$$

for all $t \geq 0$ on the event $\varepsilon_{\infty}$. Applying Cramér's and Wald's techniques, we conclude that the finite-dimensional distributions of $n^{-1} X_{[n t]}, t \geq 0$, weakly converge to the corresponding distributions of $\alpha t, t \geq 0$, on the event $\varepsilon_{\infty}$.

Now we prove the density for the distributions of $\left\{n^{-1} X_{[n t]}, t \in[0, T]\right\}, n \geq 1$. Let $s, t \in[0, T]$ and $0 \leq s<t \leq T$. Using the elementary inequality $(a+b)^{2} \leq 2\left(a^{2}+b^{2}\right)$, we obtain

$$
\begin{aligned}
\mathrm{E}\left(\frac{X_{[n t]}}{n}-\frac{X_{[n s]}}{n}\right)^{2} & =\frac{1}{n^{2}} \mathrm{E}\left(\alpha \sum_{k=[n s]+1}^{[n t]} I\left(X_{k}>0\right)+\sum_{k=[n s]+1}^{[n t]} M_{k}\right)^{2} \\
& \leq \frac{2}{n^{2}}\left(\alpha^{2}([n t]-[n s])^{2}+\sum_{k=[n s]+1}^{[n t]} \mathrm{E} M_{k}^{2}\right) \\
& \leq 3\left(\alpha^{2}(t-s)^{2}+\frac{1}{n^{2}} \sum_{k=[n s]+1}^{[n t]} \mathrm{E} X_{k} \sigma^{2}\left(X_{k}\right)\right)
\end{aligned}
$$


An argument similar to that used to prove (77) shows that

$$
\begin{aligned}
\mathrm{E}\left(n^{-1} X_{[n t]}-n^{-1} X_{[n s]}\right)^{2} & \leq 3\left(\left(\alpha^{2}(t-s)^{2}+C \frac{\alpha^{\beta}}{1+\beta} \frac{n^{1+\beta}}{n^{2}}\left(t^{1+\beta}-s^{1+\beta}\right)\right)\right. \\
& \leq 3\left(\alpha^{2}(t-s)^{2}+\frac{C(\alpha T)^{\beta}}{n^{1-\beta}}(t-s)\right)
\end{aligned}
$$

for sufficiently large $n$. Thus Theorem 12.3 of 13 implies that the distributions of $X_{[n t]} / n, t \in[0, T], n \geq 1$, are dense, since $X_{0} / n \stackrel{\mathrm{P}}{\rightarrow} 0$ as $n \rightarrow \infty$. By (8) and by the convergence criteria proposed in [13] we obtain the weak convergence in $D[0, T]$ :

$$
\frac{X_{[n t]}}{n} \rightarrow \alpha t \quad \text { as } n \rightarrow \infty
$$

on the event $\varepsilon_{\infty}$. Since the limit process is continuous, convergence (9) holds also in the uniform topology, and this completes the proof of Theorem 1]

Throughout the rest of the paper we consider the branching process $X_{k}, k \geq 0$, on the event $\varepsilon_{\infty}$ only. By the restriction method, one always can assume that $\varepsilon_{\infty}$ is the sample space. It is obvious that $X_{k} \geq 1$ for all $k \geq 0$ on this event.

We denote by $\stackrel{D}{\longrightarrow}$ the weak convergence of stochastic processes in the space $D[0, T]$; the symbol $W$ stands for a standard Wiener process.

Theorem 2. Let $m(x)=1+\alpha / x$ for some $\alpha>0$ and let $x \sigma^{2}(x)=1$. Suppose that, for all $\varepsilon>0$,

$$
\gamma_{n}(\varepsilon)=n \sup _{x \geq 1} \mathrm{E}\left(\left(\xi_{1,1}(x)-m(x)\right)^{2} I\left(\left|\xi_{1,1}(x)-m(x)\right|>\varepsilon \sqrt{n}\right)\right) \rightarrow 0
$$

as $n \rightarrow \infty$. Then

$$
\frac{X_{[n t]}-n \alpha t}{\sqrt{n}}, t \in[0, T] \stackrel{D}{\longrightarrow} W(t), t \in[0, T] \quad \text { as } n \rightarrow \infty
$$

in the space $D[0, T]$ on the event $\varepsilon_{\infty}$.

Theorem 3. Let $m(x)=1+\alpha / x$ for some $\alpha>0$ and let $x \sigma^{2}(x)=1$. Suppose there exists a nonrandom number $A>0$ such that

$$
\xi_{k, j}(x) \leq A \quad \text { a.s. }
$$

for all $k, j, x \in \mathbb{N}$. Then Theorem 2 holds.

Theorem 4. Let $m(x)=1+\alpha / x$ and $x \sigma^{2}(x)=x^{-2(1-\gamma)}$ for some $\alpha>0$ and $0<\gamma<1$. Suppose

$$
n^{2(1-\gamma)} \sup _{x \geq 1} \mathrm{E}\left(\left(\xi_{1,1}(x)-m(x)\right)^{2} \cdot I\left(\left|\xi_{1,1}(x)-m(x)\right|>\varepsilon n^{\gamma}\right)\right) \rightarrow 0 \quad \text { as } n \rightarrow \infty
$$

for every $\varepsilon>0$. Then

$$
n^{-\gamma}\left(X_{[n t]}-n \alpha t\right), t \in[0, T] \stackrel{D}{\longrightarrow} W(\rho(t)), t \in[0, T] \quad \text { as } n \rightarrow \infty
$$

in the space $D[0, T]$ on the event $\varepsilon_{\infty}$, where

$$
\rho(t)=\frac{\alpha^{2 \gamma-1}}{2 \gamma} t^{2 \gamma} .
$$

Note that $\mathrm{P}\left(\varepsilon_{\infty}\right)>0$ under the assumptions imposed on the functions $m(x)$ and $\sigma^{2}(x)$ in Theorems 24 4 (see [4).

It is easy to see that the condition of uniform boundedness for the random variables $\xi_{k, j}(x)$ in Theorem 3 implies that $\gamma_{n}(\varepsilon) \rightarrow 0$ as $n \rightarrow \infty$ in Theorem 2 Since Theorems 2 and 4 are proved similarly, we restrict ourselves to the proof of Theorem 2. 
Remark. Theorems 2 and 3 remain true if we assume that $x \sigma^{2}(x) \rightarrow 1$ as $x \rightarrow \infty$ instead of $x \sigma^{2}(x)=1$.

Proof of Theorem 2, It is easy to see that

$$
\frac{X_{[n t]}-n \alpha t}{\sqrt{n}}=\frac{1+[n t] \alpha-n \alpha t}{\sqrt{n}}+\frac{1}{\sqrt{n}} \sum_{k=1}^{[n t]} M_{k} .
$$

Since

$$
\sup _{0 \leq t \leq T} \frac{|1+[n t] \alpha-n \alpha t|}{\sqrt{n}} \rightarrow 0 \quad \text { as } n \rightarrow \infty
$$

Theorem 4.1 of 13 implies that Theorem 2 follows from

$$
\frac{1}{\sqrt{n}} \sum_{k=1}^{[n t]} M_{k}, t \in[0, T] \stackrel{D}{\longrightarrow} W(t), t \in[0, T] \quad \text { as } n \rightarrow \infty .
$$

The latter relation holds if

$$
\frac{1}{n} \sum_{k=1}^{[n t]} \mathrm{E}\left(M_{k}^{2} / \mathcal{F}_{k}\right) \rightarrow t \quad \text { as } n \rightarrow \infty
$$

and

$$
L_{n}(t)=\frac{1}{n} \sum_{k=1}^{[n t]} \mathrm{E}\left(M_{k}^{2} I\left(\left|M_{k}\right|>\varepsilon \sqrt{n}\right) / \mathcal{F}_{k}\right) \stackrel{\mathrm{P}}{\rightarrow} 0 \quad \text { as } n \rightarrow \infty
$$

for all $\varepsilon>0$ (see Theorem 7.1.11 in [12]).

Consider (12). It is clear that

$$
\frac{1}{n} \sum_{k=1}^{[n t]} \mathrm{E}\left(M_{k}^{2} / \mathcal{F}_{k}\right)=\frac{1}{n} \sum_{k=1}^{[n t]} X_{k-1} \sigma^{2}\left(X_{k-1}\right)=\frac{[n t]}{n} \rightarrow t \quad \text { as } n \rightarrow \infty,
$$

whence (12) follows.

Now we prove (13). We have

$$
\begin{aligned}
L_{n}(t)= & \frac{1}{n} \sum_{k=1}^{[n t]} \mathrm{E}\left(\sum_{i=1}^{X_{k-1}}\left(\xi_{k, i}\left(X_{k-1}\right)-m\left(X_{k-1}\right)\right)^{2} I\left(\left|M_{k}\right|>\varepsilon \sqrt{n}\right) / \mathcal{F}_{k}\right) \\
& +\frac{2}{n} \sum_{k=1}^{[n t]} \mathrm{E} \sum_{i=2}^{X_{k-1}} \sum_{j=1}^{i-1}\left(\left(\xi_{k, i}\left(X_{k-1}\right)-m\left(X_{k-1}\right)\right)\left(\xi_{k, j}\left(X_{k-1}\right)-m\left(X_{k-1}\right)\right)\right. \\
= & \left.\quad \times I\left(\left|M_{k}\right|>\varepsilon \sqrt{n}\right) / \mathcal{F}_{k}\right) \\
& L_{n 1}(t)+L_{n 2}(t) .
\end{aligned}
$$

Put

$$
S_{j}^{k}=\sum_{i=1}^{X_{k-1}}\left(\xi_{k, i}\left(X_{k-1}\right)-m\left(X_{k-1}\right)\right)-\left(\xi_{k, j}\left(X_{k-1}\right)-m\left(X_{k-1}\right)\right), \quad j \leq X_{k-1} .
$$

Using the inequality

$$
I(|X+Y|>2 \varepsilon) \leq I(|X|>\varepsilon)+I(|Y|>\varepsilon),
$$


which is true for arbitrary random variables $X, Y$ and for all $\varepsilon>0$, we get

$$
\begin{aligned}
L_{n 1}(t) \leq & \frac{1}{n} \sum_{k=1}^{[n t]} \mathrm{E}\left(\sum_{j=1}^{X_{k-1}}\left(\xi_{k, j}\left(X_{k-1}\right)-m\left(X_{k-1}\right)\right)^{2} I\left(\left|S_{j}^{k}\right|>\frac{\varepsilon \sqrt{n}}{2}\right) / \mathcal{F}_{k}\right) \\
+ & \frac{1}{n} \sum_{k=1}^{[n t]} \mathrm{E}\left(\sum_{j=1}^{X_{k-1}}\left(\xi_{k, j}\left(X_{k-1}\right)-m\left(X_{k-1}\right)\right)^{2}\right. \\
& \left.\times I\left(\left|\xi_{k, j}\left(X_{k-1}\right)-m\left(X_{k-1}\right)\right|>\frac{\varepsilon \sqrt{n}}{2}\right) / \mathcal{F}_{k}\right) \\
= & L_{n 1}^{(1)}(t)+L_{n 1}^{(2)}(t) .
\end{aligned}
$$

Since $\xi_{k, j}\left(X_{k-1}\right)-m\left(X_{k-1}\right)$ and $S_{j}^{k}$ are conditionally independent with respect to $\mathcal{F}_{k}$, we apply the second Chebyshev inequality (see [17]) and obtain

$$
\begin{aligned}
L_{n 1}^{(1)}(t) & \leq \frac{1}{n} \sum_{k=1}^{[n t]} \sum_{j=1}^{X_{k-1}} \sigma^{2}\left(X_{k-1}\right) \cdot \mathrm{P}\left(\left|S_{j}^{k}\right|>\frac{\varepsilon \sqrt{n}}{2} / \mathcal{F}_{k}\right) \\
& \leq \frac{4}{\varepsilon^{2} n^{2}} \sum_{k=1}^{[n t]} X_{k-1}\left(X_{k-1}-1\right) \sigma^{4}\left(X_{k-1}\right) \leq \frac{4[n t]}{\varepsilon^{2} n^{2}} \rightarrow 0 \quad \text { as } n \rightarrow \infty .
\end{aligned}
$$

Theorem 1 and continuous mapping theorem (Theorem 5.1 of [13]) imply that

$$
\frac{1}{n^{2}} \sum_{k=1}^{[n t]} X_{k} \rightarrow \frac{1}{n} \int_{0}^{\frac{[n t]}{n}} X_{[n s]} d s \stackrel{\mathrm{P}}{\rightarrow} \frac{\alpha t^{2}}{2} \quad \text { as } n \rightarrow \infty .
$$

Therefore,

$$
L_{n 1}^{(2)}(t) \leq \gamma_{n}\left(\frac{\varepsilon}{2}\right) \frac{1}{n^{2}} \sum_{k=1}^{[n t]} X_{k-1}=\frac{1}{n} \int_{0}^{\frac{[n t]}{n}} X_{[n s]} d s \cdot \gamma_{n}\left(\frac{\varepsilon}{2}\right) \stackrel{\mathrm{P}}{\rightarrow} 0 \quad \text { as } n \rightarrow \infty .
$$

We derive from (15)-(17) that

$$
L_{n 1}(t) \stackrel{P}{\rightarrow} 0 \quad \text { as } n \rightarrow \infty .
$$

We now pass to the estimation of $L_{n 2}(t)$. Put

$$
\theta_{k}=2 \sum_{i=2}^{X_{k-1}} \sum_{j=1}^{i-1}\left(\xi_{k, i}\left(X_{k-1}\right)-m\left(X_{k-1}\right)\right)\left(\xi_{k, j}\left(X_{k-1}\right)-m\left(X_{k-1}\right)\right) .
$$

Then

$$
L_{n 2}(t)=\frac{1}{n} \sum_{k=1}^{[n t]} \mathrm{E}\left(\theta_{k} \cdot I\left(\left|M_{k}\right|>\varepsilon \sqrt{n}\right) / \mathcal{F}_{k}\right)
$$

It is not hard to see that

$$
\mathrm{E}\left(\theta_{k}^{2} / \mathcal{F}_{k}\right)=4 \sum_{i=2}^{X_{k-1}}(i-1) \sigma^{4}\left(X_{k-1}\right)=2 X_{k-1}\left(X_{k-1}-1\right) \sigma^{4}\left(X_{k-1}\right) \leq 2 .
$$


Applying the Cauchy-Bunyakovskil inequality for conditional expectations [17, we deduce from the latter relation that

$$
\begin{aligned}
L_{n 2}(t) & \leq \frac{1}{n} \sum_{k=1}^{[n t]}\left(\mathrm{E}\left(\theta_{k}^{2} / \mathcal{F}_{k}\right)\right)^{1 / 2} \cdot\left(\mathrm{P}\left(\left|M_{k}\right|>\varepsilon \sqrt{n} / \mathcal{F}_{k}\right)\right)^{1 / 2} \\
& \leq \frac{\sqrt{2}}{n} \sum_{k=1}^{[n t]}\left(\frac{\mathrm{E}\left(M_{k}^{2} / \mathcal{F}_{k}\right)}{\varepsilon^{2} n}\right)^{1 / 2}=\frac{\sqrt{2}}{\varepsilon n^{3 / 2}} \sum_{k=1}^{[n t]}\left(X_{k-1} \cdot \sigma^{2}\left(X_{k-1}\right)\right)^{1 / 2}=\frac{\sqrt{2}[n t]}{\varepsilon n^{3 / 2}} \rightarrow 0
\end{aligned}
$$

as $n \rightarrow \infty$. This together with (14) and (18) implies (13). The proof of Theorem 2 is complete.

Example. Suppose a random variable $\xi_{k, j}(x)$ takes values 0,1 , and 2 with probabilities $\frac{1}{2} x^{-2}, 1-x^{-1}-x^{-2}$, and $x^{-1}+\frac{1}{2} x^{-2}$, respectively. In this case, $m(x)=1+x^{-1}$ and $\sigma^{2}(x)=x^{-1}$. Thus, the assumptions of Theorem 1 are satisfied with $\alpha=1$ and $\beta=0$. Hence Theorem 1 implies that the process $n^{-1} X_{[n t]}, t \in[0, T]$, weakly converges as $n \rightarrow \infty$ to the process $t, t \in[0, T]$, on the event $\varepsilon_{\infty}$. Moreover, $q<1$ by Theorem 1.4 in [3].

All the assumptions of Theorem 3 hold for this process and therefore the process $n^{-1 / 2}\left(X_{n}(t)-n t\right), t \geq 0$, weakly converges in $D[0, T]$ to the Wiener process $W(t), t \geq 0$, as $n \rightarrow \infty$.

Acknowledgement. The author is indebted to the anonymous reviewer for the valuable comments helped to improve this paper.

\section{BIBLIOGRAPHY}

1. P. Haccou, P. Jagers, and V. A. Vatutin, Branching Processes. Variation, Growth, and Extinction of Populations, Cambridge University Press, 2005. MR2429372 (2009h:92064)

2. Joshua B. Levy, Transience and recurrence of state-dependent branching processes with an immigration component, Adv. Appl. Probab. 11 (1979), 73-92. MR.517552 (80b:60111a)

3. G. Kersting, On recurrence and transience of growth models, J. Appl. Probab. 23 (1986), 614625. MR 855369 (88h:60086)

4. G. Kersting, Some properties of stochastic difference equations, Stochastic Modeling in Biology (P. Tautu, ed.), World Scientific, Singapore, 1990, pp. 328-339. MR1090847 (92c:60091)

5. P. Küster, Asymptotic growth of controlled Galton-Watson processes, Ann. Probab. 13 (1985), no. 4, 1157-1178. MR806215 (87c:60072)

6. F. C. Klebaner, A limit theorem for population-size-dependent branching processes, J. Appl. Probab. 22 (1985), 48-57. MR776887 (86h:60166)

7. F. C. Klebaner, Geometric rate of growth in population-size-dependent branching processes, J. Appl. Probab. 21 (1984), 40-49. MR732669 (85f:60124)

8. D. Pierre-Loti-Viaud, A strong law and a central limit theorem for controlled Galton-Watson processes, J. Appl. Probab. 31 (1994), 22-37. MR1260568 (94m:60175)

9. N. Lalam and C. Jacob, Estimation of the offspring mean in a supercritical or near-critical size-dependent branching process, Adv. Appl. Probab. 36 (2004), 582-601. MR2058150 (2005f:60182)

10. N. Lalam, C. Jacob, and P. Jagers, Modelling the PCR amplification process by a size-dependent branching process and estimation of the efficiency, Adv. Appl. Probab. 36 (2004), 602-615. MR 2058151 (2004m:60184)

11. A. N. Shiryaev, Probability, Nauka, Moscow, 1980; English transl., Springer-Verlag, New York, 1996. MR:1368405 (97c:60003)

12. R. Sh. Liptser and A. N. Shiryaev, Theory of Martingales, Nauka, Moscow, 1986; English transl., Kluwer Academic Publishers Group, Dordrecht, 1989. MR1022664 (90j:60046)

13. P. Billingsley, Convergence of Probability Measures, John Wiley \& Sons, Inc., New YorkLondon-Sydney, 1968. MR0233396 (38:1718)

14. B. A. Sevast'yanov and A. M. Zubkov, Controlled branching processes, Teor. Veroyatnost. i Primenen. 19 (1974), no. 1, 15-25; English transl. in Theory Probab. Appl. 19 (1974), no. 1, 11-24. MR0339350(49:4109) 
15. A. M. Zubkov, Analogies between Galton-Watson processes and $\varphi$-branching processes, Teor. Veroyatnost. i Primenen. 19 (1974), no. 2, 319-339; English transl. in Theory Probab. Appl. 19 (1974), no. 2, 309-331. MR0353480 (50:5963)

16. F. C. Klebaner, On population-size-dependent branching processes, Adv. Appl. Probab. 16 (1984), 30-55. MR732129 (85g:60090)

17. A. A. Borovkov, Probability theory, Moscow, Nauka, 1986; English transl., Gordon and Breach Science Publishers, Amsterdam, 1998. MR891184 (88c:60001)

18. T. Fujimagari, Controlled Galton-Watson process and its asymptotic behavior, Kodai Math. Sem. Rep. 27 (1976), 11-18. MR0400435 (53:4268)

19. L. V. Levina, A. M. Leontovich, and I. I. Pyatetskiǔ-Shapiro, A controllable branching process, Problemy Peredachi Informacii IV (1968), no. 2, 72-82; English transl. in Problems of Information Transmission IV (1971), no. 2, 55-64. MR0283891 (44:1121)

20. V. A. Labkovskiı̌, A limit theorem for generalized random branching processes dependent on the size of the population, Teor. Veroyatnost. i Primenen. 17 (1972), no. 1, 71-83; English transl. in Theory Probab. Appl. 17 (1972), no. 1, 72-85. MR0298785 (45:7834)

21. F. C. Klebaner, Population-size-dependent branching process with linear rate of growth, J. Appl. Probab. 20 (1983), 242-250. MR698528 (84g:60139)

Department of Probability Theory and Mathematical Statistics, Institute of Mathematics and Information Technologies, Durmon Yuli Street 29, Tashkent 100125, Uzbekistan

E-mail address: yakubjank@mail.ru

Received 6/AUG/2007

Translated by O. KLESOV 